\title{
Advanced gastric endocrine cell carcinoma with distant lymph node metastasis: a case report and clinicopathological characteristics of the disease
}

\author{
Naokazu Chiba ${ }^{1}$, Tatsushi Suwa ${ }^{2}$, Masao Hori ${ }^{3}$, Masayoshi Sakuma ${ }^{2}$, and Masaki Kitajima ${ }^{1}$ \\ ${ }^{1}$ Department of Surgery, School of Medicine, Keio University, Tokyo, Japan \\ ${ }^{2}$ Department of Surgery, Mito Red Cross Hospital, 3-12-48 Sannomaru, Mito, Ibaraki 310-0011, Japan \\ ${ }^{3}$ Department of Pathology, Mito Red Cross Hospital, Ibaraki, Japan
}

\begin{abstract}
Gastric pure endocrine cell carcinoma (ECC) is extremely rare. ECC occasionally shows multidirectional differentiation; that is, adenocarcinomatous and/or squamous proliferation. Because gastric ECC has aggressive biological behavior and shows frequent metastasis to liver and lymph nodes even in the early stage, the prognosis of patients having this disease is extremely poor. We treated a 75-year-old woman with advanced gastric pure ECC with total gastrectomy and lymph node dissection, and reviewed all the previously reported cases of this disease. We compared the clinicopathological findings of ECC with those of gastric carcinoma (GC) and found that ECC had significantly more frequent invasion to lymphatic and vascular lumens $(P<0.01)$ and more frequent metastasis to lymph nodes $(P<0.01)$ and liver $(P<0.05)$ compared to GC. Gastric ECC smaller than $5 \mathrm{~cm}$ in the greatest dimension showed a higher percentage of advanced lesions ( $>$ T2) than GC $(P<0.05)$, which could result in the difficulty of finding early ECC. The findings of the analyses we made in this report may account for the poor prognosis of this disease.
\end{abstract}

Key words Gastric endocrine cell carcinoma - Lymph node metastasis · Liver metastasis

\section{Introduction}

Extrapulmonary small-cell carcinoma is rare in the gastrointestinal tract, accounting for about $1 \%$ of cancers in the esophagus, $0.2 \%$ in the colon, and $0.1 \%-0.4 \%$ in the stomach [1-4]. Extrapulmonary small-cell carcinoma has been variously termed "endocrine cell carcinoma (ECC)", "atypical carcinoid" [5], and "oat-cell carcinoma" [6]. Worldwide, 119 cases of gastric ECC have been reported. These include pure ECC, composite type, and mixed glandular and/or squamous differen-

Offprint requests to: T. Suwa

Received: November 5, 2003 / Accepted: March 1, 2004 tiation [7]. The prognosis of gastric ECC is extremely poor, and lymph node and liver metastases are observed frequently [7]. We hypothesized that lymphatic or blood vessels incur high rates of permeation even in the early stage of gastric ECC, and this results in the frequent lymph node and liver metastases. In this study, we report our treatment of a 75-year-old woman with pure ECC. We also analyzed characteristics of the clinicopathological findings of gastric ECC by reviewing cases previously reported in the literature, and comparing the clinicopathological findings (lymphatic permeation, blood vessel permeation, lymph node metastasis, and liver metastasis) with those of gastric carcinoma, using statistical analysis.

\section{Case report}

A 75-year-old woman was referred to Mito Red Cross Hospital (Mito, Ibaraki, Japan) because of an abnormal gastric lesion first detected at a medical checkup in May 2002, at which time she had no symptoms. On admission, there was no enlargement of any superficial lymph nodes. Peripheral blood showed normal levels of all the basic biochemical parameters, and a slightly high level of the tumor marker, carcinoembryonic antigen (CEA; $6.0 \mathrm{ng} / \mathrm{ml}$; normal range $<4.0 \mathrm{ng} / \mathrm{ml}$ ). Upper gastrointestinal radiograph revealed a mass with a central ulceration, surrounded by a smooth elevated area, and with a clear margin, in the lesser curvature close to the esophagogastric junction (EGJ) (Fig. 1A). Upper gastrointestinal endoscopic study showed a 2-cm mass with a deep central depression and surrounded by a smooth elevated area, findings which corresponded to the radiographic image (Fig. 1B). The mass lesion was suspected to be ECC, based on the results of immunohistochemical staining of biopsy samples being positive for CD10 and cytokeratin (Fig. 2). Chest radiography and computerized tomographic (CT) scan of the chest did not 

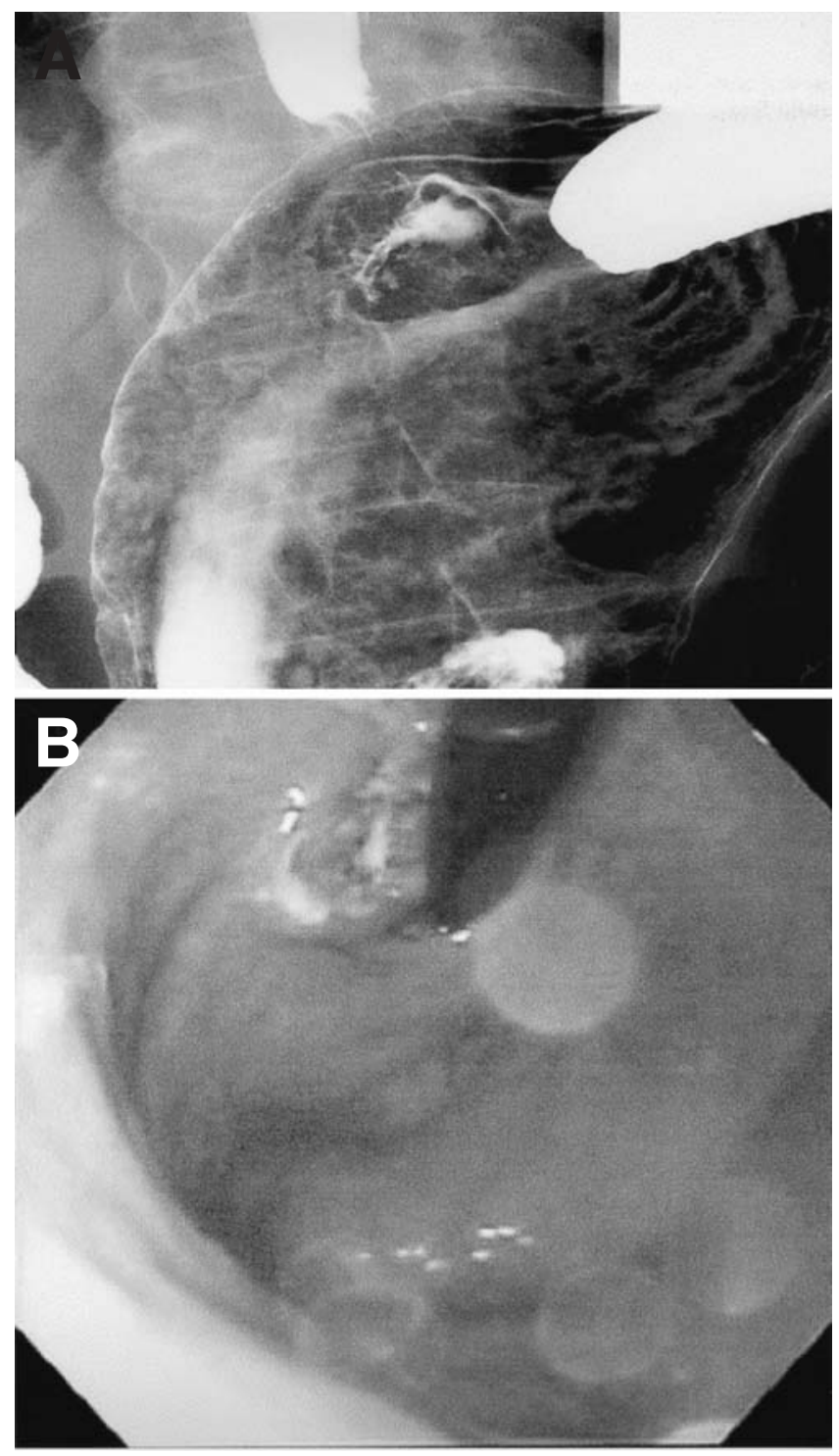

Fig. 1. A Upper gastrointestinal radiograph and B endoscopy. A Upper gastrointestinal radiograph revealed a mass, with central ulceration, surrounded by a smooth elevated area and with a clear margin, in the lesser curvature close to the esophagogastric junction. B Upper gastrointestinal endoscopy showed a mass of about $2 \mathrm{~cm}$ with a deep central depression surrounded by a smooth elevated area

show any metastatic lesions. Abdominal CT scan revealed a thickened lesion close to the EGJ and lymphadenopathy adjacent to the EGJ. No liver metastasis was found.

Total gastrectomy, splenectomy, cholecystectomy, and standard lymph node dissection were performed, without any complication, in July 2002. Macroscopic findings of the resected stomach showed a $2.0 \times 2.0-\mathrm{cm}$ tumor in the EGJ area along the lesser curvature, which had apparently invaded the muscle layer of the stomach wall. Swollen lymph nodes adjacent to the EGJ, com- mon hepatic artery, and left gastric artery were hard and were considered to be metastatic lesions.

Light-microscopically, the tumor was composed of a population of small cells with dark round nuclei and scant cytoplasm (Fig. 3A). The tumor showed invasion to a lymphatic lumen (Fig. 3B) and a vascular lumen (Fig. 3C). Numerous abnormal mitotic figures were present. There was no adenocarcinomatous or squamous proliferation, so this case was diagnosed as pure ECC. The immunohistochemical staining was intensely and diffusely positive for cytokeratin (Fig. 2B). The tumor cells were also positively stained for synaptophysin and CD56 (Fig. 2C,D). The lymph nodes adjacent to the EGJ, the common hepatic artery, and the left gastric artery were swollen and were judged to be metastatic lesions. The lymph nodes along the superior mesenteric vein were not swollen and were thought not to be metastatic during the operative procedure, but microscopically, these were seen to be the same cells as those in the tumor and were finally diagnosed as metastatic lesions. In fresh tumor material, electronmicroscopic findings showed that the neoplastic small cells with densely stained nuclei were tightly apposed and that intercellular junctions were occasionally found. Electron-dense granules (which are essential for the diagnosis of ECC), surrounded by a limiting membrane, were clearly observed (Fig. 3D). Taken together, these morphologic and immunohistochemical features confirmed the diagnosis of this case as gastric ECC.

The patient did not have any complication in her postoperative course and was discharged from the hospital, taking TS-1 (tegafur/gimeracil/oteracil potassium) orally at a dose of $100 \mathrm{mg}$ daily. At 5 months after the operation, she was examined by abdominal CT scan, which showed no recurrence in the peritoneal cavity. But she began to experience pain in the lower back from April 2003, and bone scintigraphy revealed metastatic lesions in the bilateral sacroiliac joints. She died 13 months after the operation.

\section{Discussion}

Extrapulmonary malignant neoplasms that are composed largely or entirely of small neoplastic cells with a markedly hyperchromatic nucleus and scant cytoplasm have been variously termed: "endocrine cell carcinoma (ECC)", "small cell carcinoma", "oat-cell carcinoma" [6], "neuroendocrine cell carcinoma" [8], "anaplastic carcinoma" [9], "apudoma" [10], "small cell epidermoid carcinoma" [11], and "atypical carcinoid" [5]. Extrapulmonary sites of ECC account for $4 \%$ of all cases [12], and, in particular, gastric ECC is extremely rare, accounting for $0.1 \%-0.2 \%$ of all gastric carcinomas [1-4]. 


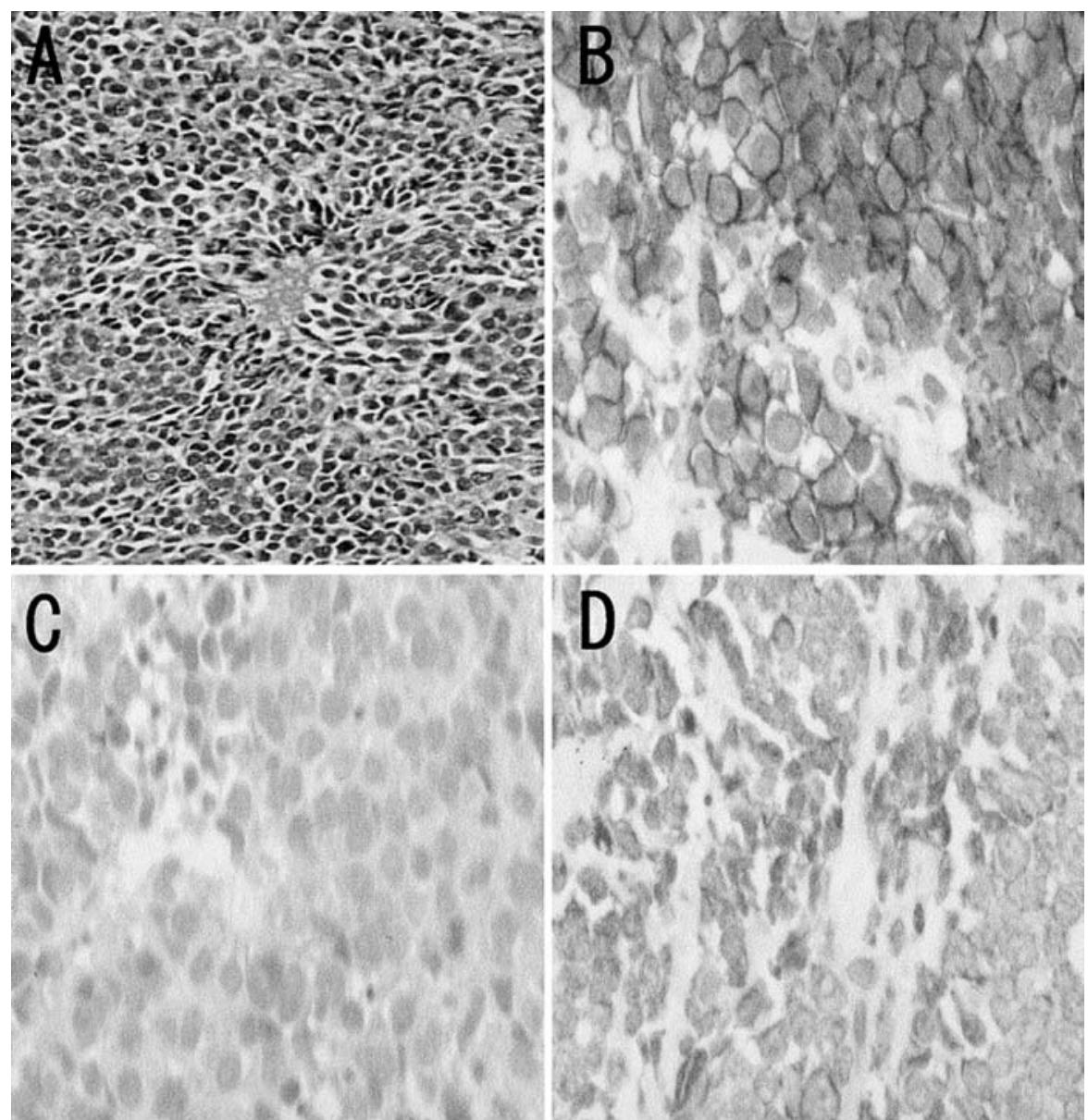

Fig. 2A-D. Histopathological findings of the biopsy samples. A The tumor was composed of small cells with densely hyperchromatic nuclei and scanty cytoplasm. B The tumor cells were stained intensely and diffusely for cytokeratin. C The tumor cells were positively stained for synaptophysin. D The tumor cells were positively stained for CD56. A H\&E, $\times 400 ; \mathbf{B} \times 400$; $\mathbf{C} \times 400 ; \mathbf{D} \times 400$
We employ the term "endocrine cell carcinoma (ECC)", because the term "small" is not clearly defined in extrapulmonary lesions, and this neoplasm is believed to originate from endodermally derived stem cells $[13,14]$. Aggressive or exclusive proliferation of small neoplastic cells was evident even in the early stage of gastric ECC in the invaded lymphatic and/or vascular lumens, and also metastatic sites; in particular, in the regional lymph nodes and liver [7].

To date, 119 cases of gastric ECC have been reported in the literature, in terms of the clinicopathological findings, therapies, and/or prognosis of gastric ECC. The mean age at presentation was 64.9 years (range, $31-$ 89 years). From the data given, 87 of the patients with the tumors were men and 31 were women. Forty-one tumors arose in the antrum of the stomach, 32 in the middle, 35 in the cardia, and 6 in the stump. The size of the tumor at its greatest dimension ranged from 0.4 to $16.0 \mathrm{~cm}$ (mean, $6.3 \mathrm{~cm}$ ). The $\mathrm{T}$ factor in the TNM grading system was $\mathrm{T} 1$ in 13 patients, $\mathrm{T} 2$ in 43 patients, $\mathrm{T} 3$ in 23 patients, and T4 in 21 patients. Forty-one tumors were showed invasion to lymphatic lumens, and 34 to vascular lumens. Seventy-eight patients had metastatic sites in regional lymph nodes, and 27 patients had them in the liver. The disease was stage I in 11 patients, stage II in 15 patients, stage III in 25 patients, and stage IV in 46 patients. We compared the clinicopathological features of all the reported ECC cases with those of 10000 gastric carcinoma (GCs) reported by Nakajima et al. [15] and those of about 5000 GCs reported by the Japanese Gastric Cancer Association [16]. The results showed that ECC had more frequent invasion to the lymphatic lumen $(88.9 \%$ vs $56.6 \% ; P<0.01$; Fig. 4 A) and vascular lumen (75.6\% vs $31.6 \% ; P<0.01$; Fig. 4B). ECC also had more frequent metastasis to lymph nodes ( $82.1 \%$ vs $58.8 \%, P<0.01$; Fig. 4 C) and liver ( $26.7 \%$ vs $3.9 \% ; P<0.05$; Fig. 5A) than GC. We found that T1-2 tumors of ECC metastasized to lymph nodes more frequently compared to those of GC $(73.5 \%$ vs $41.2 \% ; P<$ 0.001; Fig. 4D), and T1 tumors also showed a difference in lymph node metastasis between ECC and GC (58.3\% vs $11.8 \% ; P<0.01$, data not shown). T2 tumors of ECC metastasized to the liver more frequently compared to those of GC (22.5\% vs $4.6 \% ; P<0.05$; Fig. 5B). T3-4 tumors, which are more advanced, than T1-2, showed no difference in lymph node or liver metastasis between 

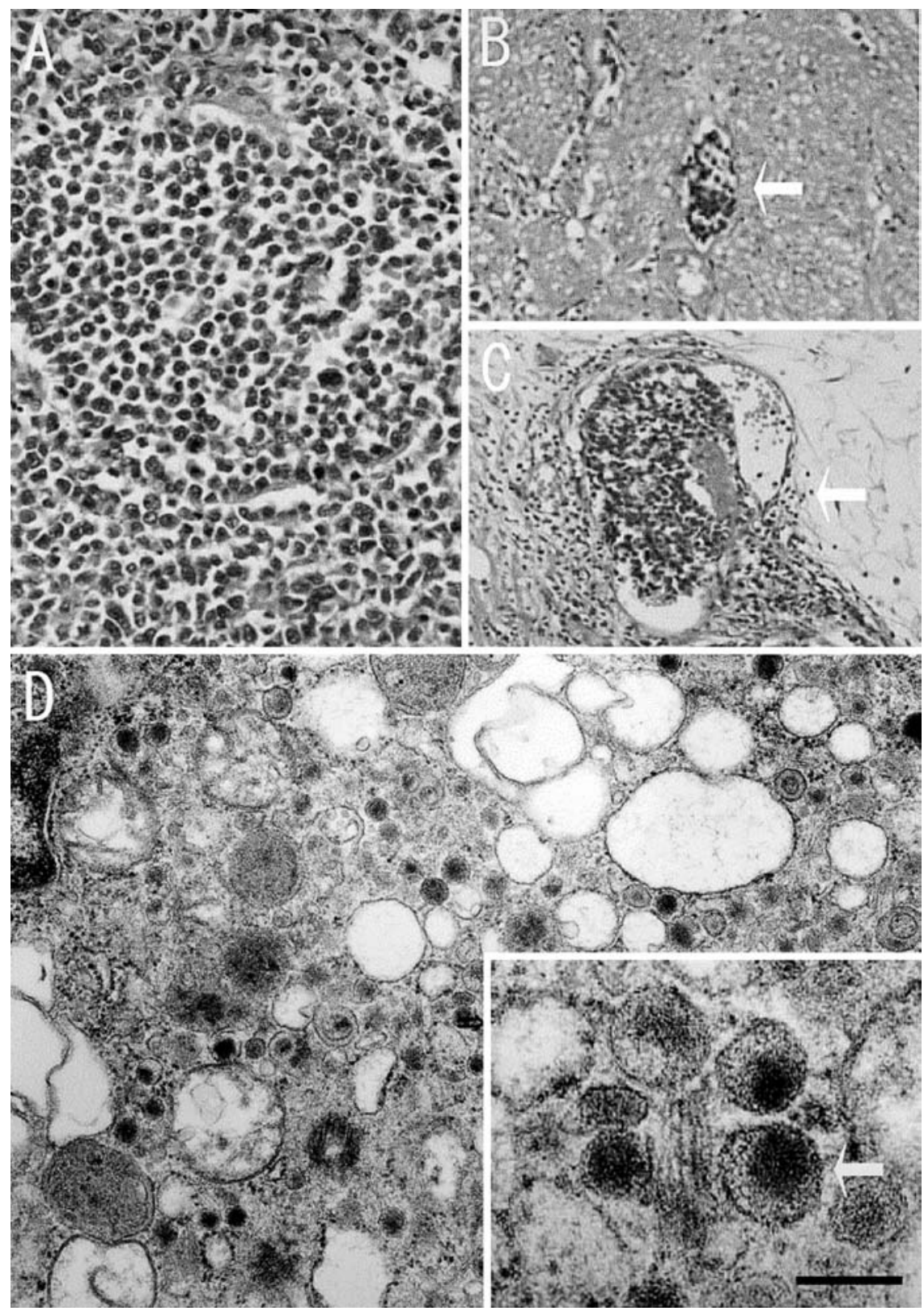

Fig. 3A-D. Histopathological findings of the resected tumor. A Lightmicroscopically, the tumor was composed of a population of small cells with dark round nuclei and scant cytoplasm. B The arrow shows tumor invasion to a lymphatic lumen. C The arrow shows tumor invasion to a vascular lumen. D Electronmicroscopically, the neoplastic small cells with densely stained nuclei are tightly apposed. Electron-dense granules surrounded by a limiting membrane are shown. Inset, higher magnification view of the granules. The arrow shows the granules. The bar represents $100 \mathrm{~nm}$. A-C $\mathrm{H} \& \mathrm{E}, \times 400 ; \mathbf{D} \times 23000$; inset in $\mathbf{D}$ $\times 46000$
ECC and GC. These results show that gastric ECC invades lymphatic and vascular lumens and frequently metastasizes to lymph nodes and liver even in the early stage. In our patient, the lymph nodes adjacent to the superior mesenteric vein were not swollen, but were microscopically diagnosed as metastatic lesions. The lymph node metastasis along the superior mesenteric vein was classified as distant metastasis (UICC $5^{\text {th }}$ edition; stomach (ICD-0 C16); 1997), which resulted in the clinical stage in this patient being diagnosed as stage IV.

Analysis of the relationship between $\mathrm{T}$ factor and tumor size at the greatest dimension revealed that ECC tumors of $0-2.0 \mathrm{~cm}$ and $2.1-5.0 \mathrm{~cm}$ in size showed T2 at a greater frequency and had a higher stage compared to
GC $(0-2.0 \mathrm{~cm}, 55.6 \%$ vs $9.7 \% ; P<0.02$; Fig. $5 \mathrm{C} ; 2.1-$ $5.0 \mathrm{~cm}, 80.0 \%$ vs $55.5 \% ; P<0.05$; Fig. 5D). These results correspond to the report that early gastric ECC is usually observed as a polypoid lesion arising from deep mucosa or submucosa, while later, a crater-like ulceration develops, most likely due to its rapid proliferation [1]. For this reason, finding an early ECC (such as T1 stage) may be very difficult compared to GC.

Gastric ECC shows variable histological patterns [7]. In the reported cases, 43 were pure ECC and 46 were tumors combined with adenocarcinoma and/or squamous cell carcinoma. The present case was a pure ECC type. Ultrastructual and biochemical studies of gastric ECC revealed the origin to be from neuroendocrine 


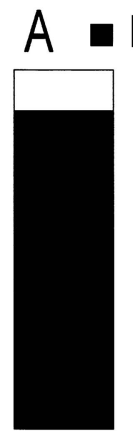

$\operatorname{ly}(+) \square \operatorname{ly}(-)$

ECC Iy $(+): 88.9 \%$ *

$(40 / 45)$

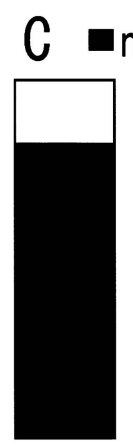

ECC

$\mathrm{n}(+): 82.1 \%{ }^{*}$

$(78 / 95)$

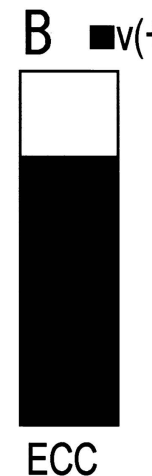

$v(+): 75.6 \%$ *

$(34 / 45)$

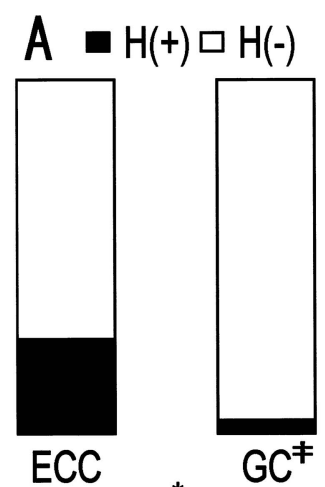

$H(+): 26.7 \%$ * $H(+): 3.9 \%$

$(27 / 101)$

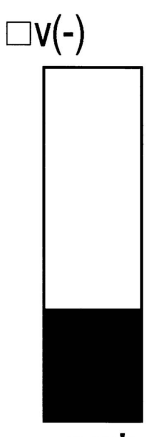

$v(+): 31.6 \%$

$(1719 / 5444)$

\section{D $\square \mathrm{n}(+) \square \mathrm{n}(-)$}

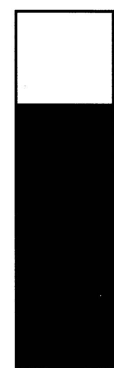

ECC

$\mathrm{n}(+): 73.5 \%{ }^{*}$

$(36 / 49)$

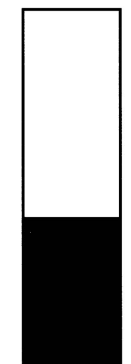

$\mathrm{GC}^{\ddagger}$

$\mathrm{n}(+): 41.2 \%$

(1926/4674)

Fig. 4A-D. Analysis of clinicopathological features of endocrine cell carcinoma (ECC) compared to gastric carcinoma $(G C)$. A ECC had more frequent invasion to lymphatic (ly) lumens compared to GC $(88.9 \%$ vs $56.6 \%$; $* P<0.01)$. B ECC had more frequent invasion to vascular $(v)$ lumens compared to $\mathrm{GC}(75.6 \%$ vs $31.6 \%$; $P<0.01)$. $\mathbf{C}$ ECC had more frequent lymph node $(n)$ metastasis $(P<0.01)$ compared to GC $(82.1 \%$ vs $58.8 \%$; $* P<0.01)$. D T1-2 tumors of ECC metastasized to lymph nodes $(n)$ more frequently than those of GC $(73.5 \%$ vs $41.2 \%$; $* P<0.001)$. ${ }^{\dagger}$ Database of about 5000 GCs (Japanese Gastric Cancer Association [16]). ${ }^{\text {DDatabase of }}$ 10000 GCs (Nakajima et al. [15])

APUD (amino precursor uptake and decarboxylation) cells, which are widely distributed along the gastrointestinal tract $[17,18]$. At present, the presence of cytoplasmic argyrophilia and structural neurosecretory granules helps in the diagnosis of gastric ECC, but it has also been reported that several tumors had no such features $[7,13,14,18]$. Therefore, immunohistochemically positive staining for several neuroendocrine markers chromogranin A, synaptophysin, synaptic vesicle protein, Leu 7 (CD57), neuron-specific enolase (NSE), protein gene product (PGP 9.5), neural cell adhesion molecule (NCAM; CD56), and cytokeratin - is helpful in determining the histology of ECC, in contrast to negative staining for CEA [7,19]. We realize that preoperative diagnosis using these immunohistochemical
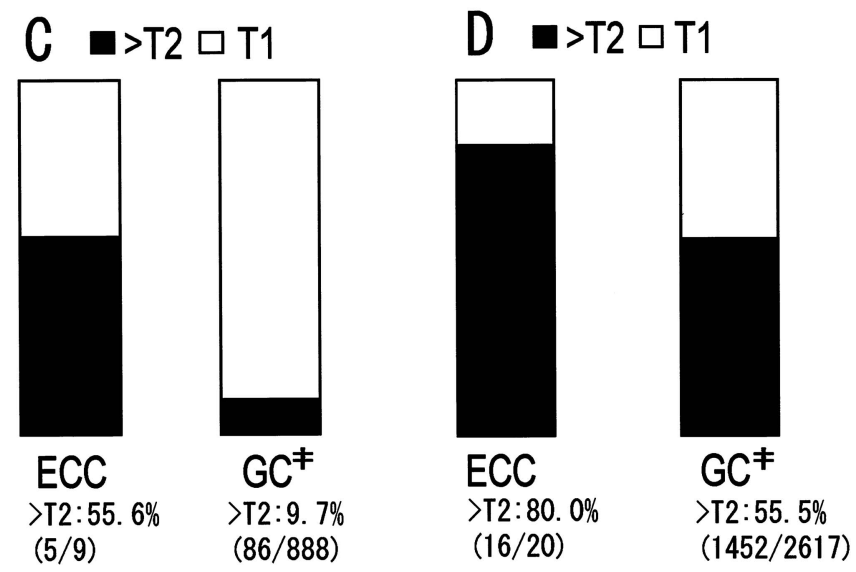

Fig. 5A-D. Analysis of clinicopathological features of endocrine cell carcinoma $(E C C)$ compared to gastric carcinoma $(G C)$. A ECC had more frequent liver metastasis $(H+)$ compared to GC $(26.7 \%$ vs $3.9 \%$; $* P<0.05)$. B T2 tumors of ECC metastasized to liver $(H+)$ more frequently than those of GC $(22.5 \%$ vs $4.6 \%$; $* P<0.05)$. C Tumors of ECC ranging from 0 to $2.0 \mathrm{~cm}$ showed T2 more frequently and were of higher stage compared to those of GC $(55.6 \%$ vs $9.7 \% ; * P<0.02)$. D Tumors of ECC ranging from 2.1 to $5.0 \mathrm{~cm}$ showed T2 more frequently and were of higher stage compared to those of GC (80.0\% vs $55.5 \%$; $* P<0.05)$. ${ }^{*}$ Database of 10000 gastric carcinomas (Nakajima et al. [15])

markers is important for gastric ECC, because its aggressive biological behavior toward distant lymph nodes is occasionally beyond our ability to detect, as occurred in our patient.

The prognosis of gastric ECC is reportedly extremely poor [7]. In the reported cases, 66 patient had 6-month survival; 42 patients, 1-year survival; and 6 patients, 5 -year survival. Concerning the treatment for gastric ECC, intensive chemotherapy with or without operation is recommended at any stage [20].

In conclusion, ECC had more frequent invasion to lymphatic and vascular lumens, and more frequent metastasis to lymph nodes and liver compared to GC. This may account for the poor prognosis of this disease. 


\section{References}

1. Matsubayashi H, Takagaki S, Otsubo T, Iiri T, Kobayashi Y, Yokota T, et al. Advanced gastric glandular-endocrine cell carcinoma with 1-year survival after gastrectomy. Gastric Cancer 2000; 3:226-33.

2. Jass JR, Sobin LH, Watanabe H. The World Health Organization's histologic classification of gastrointestinal tumors. A commentary on the second edition. Cancer 1990;66:21627.

3. Nobin A, Ahren B, Ahlman H. Endocrine tumors in the gastrointestinal tract. Nord Med 1988;103:12-4.

4. Sato T, Mukai M, Ando N, Tashiro Y, Iri H, Abe O, et al. Small cell carcinoma (non-oat cell type) of the esophagus concomitant with invasive squamous cell carcinoma and carcinoma in situ. A case report. Cancer 1986;57:328-32.

5. Sweeney EC, McDonnell LM. Atypical gastric carcinoids. Histopathology 1980;4:215-24.

6. Ibrahim NB, Briggs JC, Corbishley CM. Extrapulmonary oat cell carcinoma. Cancer 1984;54:1645-61.

7. Matsui K, Kitagawa M, Miwa A, Kuroda Y, Tsuji M. Small cell carcinoma of the stomach: a clinicopathologic study of 17 cases. Am J Gastroenterol 1991;86:1167-75.

8. Chejfec G, Gould VE. Malignant gastric neuroendocrinomas. Ultrastructural and biochemical characterization of their secretory activity. Hum Pathol 1977;8:433-40.

9. Matsusaka T, Watanabe H, Enjoji M. Anaplastic carcinoma of the esophagus. Report of three cases and their histogenetic consideration. Cancer 1976;37:1352-8.

10. Tateishi R, Wada A, Hayakawa K, Hongo J, Ishii S. Argyrophil cell carcinomas (apudomas) of the uterine cervix. Light and elec- tron microscopic observations of five cases. Virchows Arch A Pathol Anat Histopathol 1975;366:257-74.

11. Mills SE, Allen MS Jr, Cohen AR. Small-cell undifferentiated carcinoma of the colon. A clinicopathological study of five cases and their association with colonic adenomas. Am J Surg Pathol 1983;7:643-51.

12. Levenson RM Jr, Ihde DC, Matthews MJ, Cohen MH, Gazdar AF, Bunn PA Jr, et al. Small cell carcinoma presenting as an extrapulmonary neoplasm: sites of origin and response to chemotherapy. J Natl Cancer Inst 1981;67:607-12.

13. Matsuyama M, Suzuki H. Differentiation of immature mucous cells into parietal, argyrophil, and chief cells in stomach grafts. Science 1970;169:385-7.

14. Sidhu GS. The endodermal origin of digestive and respiratory tract APUD cells. Histopathologic evidence and a review of the literature. Am J Pathol 1979;96:5-20.

15. Nakajima T. Data from 10000 gastric carcinomas. Jpn J Cancer Chemother 1994;21:1813-97.

16. Japanese Gastric Cancer Association. Data from gastric carcinomas in 1997. 1997.

17. Pearse AG. Common cytochemical and ultrastructural characteristics of cells producing polypeptide hormones (the APUD series) and their relevance to thyroid and ultimobranchial $\mathrm{C}$ cells and calcitonin. Proc R Soc Lond B Biol Sci 1968;170:71-80.

18. Pearse AG, Takor TT. Neuroendocrine embryology and the APUD concept. Clin Endocrinol (Oxf) 1976;5(Suppl):229-44.

19. Sakaki M, Sano T, Hirokawa M, Takahashi M, Kiyoku H. Immunohistochemical study of endocrine cells in ductal adenocarcinoma of the pancreas. Virchows Arch 2002;441:249-55.

20. Fukuda T, Ohnishi Y, Nishimaki T, Ohtani H, Tachikawa S. Early gastric cancer of the small cell type. Am J Gastroenterol 1988;83: 1176-9. 